\title{
Existence results of nonlocal Robin mixed Hahn and $q$-difference boundary value problems
}

\section{Thongchai Dumrongpokaphan ${ }^{1}$, Nichaphat Patanarapeelert ${ }^{2}$ and Thanin Sitthiwirattham ${ }^{3 *}$ (B)}

\section{"Correspondence:}

thanin_sit@dusit.ac.th

${ }^{3}$ Mathematics Department, Faculty

of Science and Technology, Suan

Dusit University, Bangkok, Thailand

Full list of author information is

available at the end of the article

\section{Springer}

\begin{abstract}
In this paper, we aim to study a nonlocal Robin boundary value problem for fractional sequential fractional Hahn-q-equation. The existence and uniqueness results for this problem are revealed by using the Banach fixed point theorem. In addition, the existence of at least one solution is studied by using Schauder's fixed point theorem. The theorems for existence results are obtained.

MSC: 39A10; 39A13; 39A70

Keywords: Fractional q-calculus; Fractional Hahn calculus; Robin boundary value problems; Existence
\end{abstract}

\section{Introduction}

The quantum difference operator has been applied in many mathematical areas such as orthogonal polynomials, combinatorics, and the calculus of variations [1-4]. The research works related to the quantum difference operator have been published continuously.

$q$-difference operator is the one type of quantum calculus proposed by Jackson [1] which is defined by

$$
D_{q} f(t):= \begin{cases}\frac{f(q t)-f(t)}{t(q-1)}, & t \neq 0, \\ f^{\prime}(0), & t=0,\end{cases}
$$

where $q \in(0,1)$. For fractional $q$-difference operator, it was proposed by Al-Salam [5] and Agarwal [6]. Basic knowledge of fractional $q$-difference calculus can be found in [7] and [8]. The studies of $q$-difference operator can be found in [9-33].

Hahn difference operator proposed by Hahn [34] is another tool that can be employed to study families of orthogonal polynomials and approximation problems (see [35-37]) which is defined by

$$
D_{q, \omega} f(t):= \begin{cases}\frac{f(q t+\omega)-f(t)}{t(q-1)+\omega}, & t \neq \omega_{0}, \\ f^{\prime}\left(\omega_{0}\right), & t=\omega_{0},\end{cases}
$$

(c) The Author(s) 2020. This article is licensed under a Creative Commons Attribution 4.0 International License, which permits use, sharing, adaptation, distribution and reproduction in any medium or format, as long as you give appropriate credit to the original author(s) and the source, provide a link to the Creative Commons licence, and indicate if changes were made. The images or other third party material in this article are included in the article's Creative Commons licence, unless indicated otherwise in a credit line to the material. If material is not included in the article's Creative Commons licence and your intended use is not permitted by statutory regulation or exceeds the permitted use, you will need to obtain permission directly from the copyright holder. To view a copy of this licence, visit http://creativecommons.org/licenses/by/4.0/. 
where $q \in(0,1), \omega>0$, and $\omega_{0}:=\frac{\omega}{1-q}$. The right inverse operator of Hahn's operator, which generalizes both the Nörlund sum and the Jackson $q$-integral, was proposed by Aldwoah [38, 39]. The new extensive results of Hahn difference operator can be seen in [40-47]. Recently, Brikshavana et al. [48] and Wang et al. [49] introduced the fractional Hahn difference operator. The studies of fractional Hahn difference calculus can be found in [5056].

We observe that the study of the boundary value problem of mixed difference operators had not been studied until the work of Dumrongpokaphan et al. [57]. They studied sequential fractional $q$-Hahn-difference equation. In this paper, we propose a sequential fractional Hahn-q-difference equation where the difference operators are reverse. Our problem is a nonlocal Robin boundary value problem for sequential fractional Hahn-qdifference equation of the form

$$
\begin{aligned}
& D_{q, \omega}^{\alpha} D_{q}^{\beta} u(t)=F\left[t, u(t), D_{q}^{\theta} u(t), D_{q, \omega}^{v} u(t)\right], \quad t \in I_{q, \omega}^{I_{q}^{[0, T]}}, \\
& \lambda_{1} u(\eta)+\lambda_{2} D_{q}^{\gamma} u(\eta)=\phi_{1}(u), \quad \eta \in(0, T), \\
& \mu_{1} u(T)+\mu_{2} D_{q, \omega}^{\gamma} u(T)=\phi_{2}(u),
\end{aligned}
$$

where $I_{q, \omega}^{I_{q}^{[0, T]}}:=\bigcup_{k=0}^{\infty} I_{q, \omega}^{q^{k_{s}}}, s \in[0, T] ; I_{q, \omega}^{x}:=\left\{q^{n} x+\omega[n]_{q}: n \in \mathbb{N}_{0}\right\} \cup\left\{\omega_{0}\right\} ; \mathbb{N}_{0}:=\mathbb{N} \cup\{0\} ;$ $[n]_{q}:=\frac{1-q^{n}}{1-q} ; 0<q<1 ; \omega>0 ; T>\omega_{0} ; \alpha, \beta, \gamma, \theta, v \in(0,1] ; \alpha+\beta \in(1,2] ; \lambda_{1}, \lambda_{2}, \mu_{1}, \mu_{2} \in \mathbb{R}^{+}$; $F \in C([0, T] \times \mathbb{R} \times \mathbb{R} \times \mathbb{R}, \mathbb{R})$ is a given function; and $\phi_{1}, \phi_{2}: C([0, T], \mathbb{R}) \rightarrow \mathbb{R}$ are given functionals.

We organize the paper as follows. In Sect. 2, we provide some basic knowledge. In Sect. 3, we prove the existence and uniqueness of a solution to problem (1.1) by using the Banach fixed point theorem. In Sect. 4, we prove the existence of at least one solution to problem (1.1) by using Schauder's fixed point theorem. Finally, in the last section, we provide an example to show applications of our results.

\section{Preliminaries}

In this section, we recall some notations, definitions, and lemmas used in the main results.

For $q \in(0,1), \omega>0, n \in \mathbb{N}_{0}$, and $a, b \in \mathbb{R}$, we define the $q$-analogue of the power function $(a-b) \frac{n}{q}$ as

$$
(a-b) \frac{0}{q}:=1, \quad(a-b) \frac{n}{q}:=\prod_{k=0}^{n-1}\left(a-b q^{k}\right),
$$

and the $q, \omega$-analogue of the power function $(a-b) \frac{n}{q, \omega}$ as

$$
(a-b) \frac{0}{q, \omega}:=1, \quad(a-b) \frac{n}{q, \omega}:=\prod_{k=0}^{n-1}\left[a-\left(b q^{k}+\omega[k]_{q}\right)\right] .
$$

For $\alpha \in \mathbb{R}$, we define

$$
(a-b) \frac{\alpha}{q}=a^{\alpha} \prod_{n=0}^{\infty} \frac{1-\left(\frac{b}{a}\right) q^{n}}{1-\left(\frac{b}{a}\right) q^{\alpha+n}}, \quad a \neq 0,
$$




$$
(a-b) \frac{\alpha}{q, \omega}=\left(a-\omega_{0}\right)^{\alpha} \prod_{n=0}^{\infty} \frac{1-\left(\frac{b-\omega_{0}}{a-\omega_{0}}\right) q^{n}}{1-\left(\frac{b-\omega_{0}}{a-\omega_{0}}\right) q^{\alpha+n}}=\left(\left(a-\omega_{0}\right)-\left(b-\omega_{0}\right)\right)_{q}^{\frac{\alpha}{q}} \quad a \neq \omega_{0} .
$$

In addition, we define $a_{q}^{\frac{\alpha}{q}}=a^{\alpha}$ and $\left(a-\omega_{0}\right) \frac{\alpha}{q, \omega}=\left(a-\omega_{0}\right)^{\alpha}$. For $\alpha>0$, we let $(0)^{\frac{\alpha}{q}}=\left(\omega_{0}\right) \frac{\alpha}{q, \omega}=0$.

For $k \in \mathbb{N}$, the $q$-analogue and $q, \omega$-analogue of forward jump operator [58] are defined by

$$
\sigma_{q}^{k}(t):=q^{k} t \quad \text { and } \quad \sigma_{q, \omega}^{k}(t):=q^{k} t+\omega[k]_{q}
$$

respectively. The $q$-analogue and $q, \omega$-analogue of backward jump operator are defined by

$$
\rho_{q}^{k}(t):=\frac{t}{q^{k}} \quad \text { and } \quad \rho_{q, \omega}^{k}(t):=\frac{t-\omega[k]_{q}}{q^{k}}
$$

respectively.

Definition 2.1 For $q \in(0,1)$, the $q$-derivative of a real function $f$ is defined by

$$
D_{q} f(t)=\frac{f(t)-f(q t)}{(1-q) t} \quad \text { and } \quad D_{q} f(0)=f^{\prime}(0) \text {. }
$$

The $q$-integral of a function $f$ defined on the interval $[0, T]$ is defined by

$$
\mathcal{I}_{q} f(t)=\int_{0}^{t} f(s) d_{q} s=(1-q) t \sum_{n=0}^{\infty} q^{n} f\left(q^{n} t\right),
$$

where the infinite series is convergent.

Definition 2.2 For $q \in(0,1), \omega>0$, and $f$ defined on an interval $I \subseteq \mathbb{R}$ containing $\omega_{0}:=$ $\frac{\omega}{1-q}$, the Hahn difference of $f$ is defined by

$$
D_{q, \omega} f(t)=\frac{f(q t+\omega)-f(t)}{t(q-1)+\omega} \quad \text { for } t \neq \omega_{0}
$$

and $D_{q, \omega} f\left(\omega_{0}\right)=f^{\prime}\left(\omega_{0}\right)$ whenever $f$ is differentiable at $\omega_{0}$.

For $a, b \in I \subseteq \mathbb{R}, a<\omega_{0}<b$, and $[k]_{q}=\frac{1-q^{k}}{1-q}, k \in \mathbb{N}_{0}:=\mathbb{N} \cup\{0\}$, we define the $q$, $\omega$-interval by

$$
\begin{aligned}
{[a, b]_{q, \omega} } & :=\left\{q^{k} a+\omega[k]_{q}: k \in \mathbb{N}_{0}\right\} \cup\left\{q^{k} b+\omega[k]_{q}: k \in \mathbb{N}_{0}\right\} \cup\left\{\omega_{0}\right\} \\
& =\left[a, \omega_{0}\right]_{q, \omega} \cup\left[\omega_{0}, b\right]_{q, \omega} \\
& =(a, b)_{q, \omega} \cup\{a, b\}=[a, b)_{q, \omega} \cup\{b\}=(a, b]_{q, \omega} \cup\{a\} .
\end{aligned}
$$

For each $s \in[a, b]_{q, \omega}$, the sequence $\left\{\sigma_{q, \omega}^{k}(s)\right\}_{k=0}^{\infty}=\left\{q^{k} s+\omega[k]_{q}\right\}_{k=0}^{\infty}$ is uniformly convergent to $\omega_{0}$. 
Definition 2.3 Let $I$ be any closed interval of $\mathbb{R}$ containing $a, b$, and $\omega_{0}$, and $f: I \rightarrow \mathbb{R}$ is a given function. We define $q, \omega$-integral of $f$ from $a$ to $b$ by

$$
\int_{a}^{b} f(t) d_{q, \omega} t:=\int_{\omega_{0}}^{b} f(t) d_{q, \omega} t-\int_{\omega_{0}}^{a} f(t) d_{q, \omega} t
$$

where $\int_{\omega_{0}}^{x} f(t) d_{q, \omega} t:=[x(1-q)-\omega] \sum_{k=0}^{\infty} q^{k} f\left(x q^{k}+\omega[k]_{q}\right), x \in I$, and the series converges at $x=a$ and $x=b$. The sum to the right-hand side of the above equation is called the Jackson-Nörlund sum.

We note that the actual domain of function $f$ is $[a, b]_{q, \omega} \subset I$.

In what follows, we define fractional $q$-integral, fractional Hahn integral, fractional $q$ difference, and fractional Hahn difference of Riemann-Liouville type.

Definition 2.4 For $\alpha \geq 0$ and $f$ defined on $[0, T]$, the fractional $q$-integral of RiemannLiouville type is defined by

$$
\begin{aligned}
\left(\mathcal{I}_{q}^{\alpha} f\right)(t) & :=\frac{1}{\Gamma_{q}(\alpha)} \int_{0}^{t}(t-q s) \frac{\alpha-1}{q} f(t) d_{q} s \\
& =\frac{t(1-q)}{\Gamma_{q}(\alpha)} \sum_{n=0}^{\infty} q^{n}\left(t-q^{n+1} t\right)_{q}^{\frac{\alpha-1}{q}} f\left(q^{n} t\right) \\
& =\frac{t^{\alpha}(1-q)}{\Gamma_{q}(\alpha)} \sum_{n=0}^{\infty} q^{n}\left(1-q^{n+1}\right)^{\frac{\alpha-1}{q}} f\left(q^{n} t\right),
\end{aligned}
$$

and $\left(\mathcal{I}_{q}^{0} f\right)(x)=f(x)$

Definition 2.5 For $\alpha, \omega>0, q \in(0,1)$, and $f$ defined on $\left[\omega_{0}, T\right]_{q, \omega}$, the fractional Hahn integral is defined by

$$
\begin{aligned}
\mathcal{I}_{q, \omega}^{\alpha} f(t) & :=\frac{1}{\Gamma_{q}(\alpha)} \int_{\omega_{0}}^{t}\left(t-\sigma_{q, \omega}(s)\right)_{q, \omega}^{\alpha-1} f(s) d_{q, \omega} s \\
& =\frac{[t(1-q)-\omega]}{\Gamma_{q}(\alpha)} \sum_{n=0}^{\infty} q^{n}\left(t-\sigma_{q, \omega}^{n+1}(t)\right) \frac{\alpha-1}{q, \omega} f\left(\sigma_{q, \omega}^{n}(t)\right) \\
& =\frac{(1-q)\left(t-\omega_{0}\right)^{\alpha}}{\Gamma_{q}(\alpha)} \sum_{n=0}^{\infty} q^{n}\left(1-q^{n+1}\right)_{q}^{\frac{\alpha-1}{}} f\left(\sigma_{q, \omega}^{n}(t)\right),
\end{aligned}
$$

and $\left(\mathcal{I}_{q, \omega}^{0} f\right)(t)=f(t)$.

Definition 2.6 For $N \in \mathbb{N}, \alpha \geq 0$, where $N-1<\alpha \leq N$, and $f$ defined on $[0, T]$, the fractional q-derivative of the Riemann-Liouville type of order $\alpha$ is defined by

$$
\begin{aligned}
\left(D_{q}^{\alpha} f\right)(t) & :=\left(D_{q}^{N} \mathcal{I}_{q}^{N-\alpha} f\right)(t) \\
& =\frac{1}{\Gamma_{q}(-\alpha)} \int_{0}^{t}\left(t-\sigma_{q}(s)\right)_{q}^{-\alpha-1} f(s) d_{q} s,
\end{aligned}
$$

and $\left(D_{q}^{0} f\right)(x)=f(x)$. 
Definition 2.7 For $N \in \mathbb{N}, \alpha \geq 0$, where $N-1<\alpha \leq N, q \in(0,1), \omega>0$, and $f$ defined on $\left[\omega_{0}, T\right]_{q, \omega}$, the fractional Hahn difference of Riemann-Liouville type of order $\alpha$ is defined by

$$
\begin{aligned}
D_{q, \omega}^{\alpha} f(t) & :=\left(D_{q, \omega}^{N} \mathcal{I}_{q, \omega}^{N-\alpha} f\right)(t) \\
& =\frac{1}{\Gamma_{q}(-\alpha)} \int_{\omega_{0}}^{t}\left(t-\sigma_{q, \omega}(s)\right)_{q, \omega}^{\frac{-\alpha-1}{f}} f(s) d_{q, \omega} s,
\end{aligned}
$$

and $D_{q, \omega}^{0} f(t)=f(t)$.

Lemma 2.1 ([17]) For $N \in \mathbb{N}, \alpha \geq 0$, where $N-1<\alpha \leq N, q \in(0,1)$, and $f: I_{q}^{T} \rightarrow \mathbb{R}$,

$$
\mathcal{I}_{q}^{\alpha} D_{q}^{\alpha} f(t)=f(t)+C_{1} t^{\alpha-1}+\cdots+C_{N} t^{\alpha-N}
$$

for some $C_{i} \in \mathbb{R}, i=\{1,2, \ldots, N\}$.

Lemma $2.2([48])$ For $N \in \mathbb{N}, \alpha \geq 0$, where $N-1<\alpha \leq N, q \in(0,1), \omega>0$, and $f: I_{q, \omega}^{T} \rightarrow$ $\mathbb{R}$,

$$
\mathcal{I}_{q, \omega}^{\alpha} D_{q, \omega}^{\alpha} f(t)=f(t)+C_{1}\left(t-\omega_{0}\right)^{\alpha-1}+\cdots+C_{N}\left(t-\omega_{0}\right)^{\alpha-N}
$$

for some $C_{i} \in \mathbb{R}, i=\{1,2, \ldots, N\}$.

The $q$-gamma and $q$-beta functions are defined by

$$
\begin{aligned}
& \Gamma_{q}(x):=\frac{(1-q) \frac{x-1}{q}}{(1-q)^{x-1}}, \quad x \in \mathbb{R} \backslash\{0,-1,-2, \ldots\}, \\
& B_{q}(x, s):=\int_{0}^{1} t^{x-1}(1-q t) \frac{s-1}{q} d_{q} t=\frac{\Gamma_{q}(x) \Gamma_{q}(s)}{\Gamma_{q}(x+s)}, \quad \text { respectively. }
\end{aligned}
$$

Next, we aim to find a solution of the linear variant of mixed problem (1.1) where the following auxiliary lemmas will be used for simplifying calculations.

Lemma 2.3 ([21]) Let $\alpha, \beta \geq 0$ and $0<p, q<1$. Then the following formulas hold:

$$
\begin{aligned}
& \int_{0}^{\eta}(\eta-q t) \frac{\alpha-1}{q} t^{\beta} d_{q} t=\eta^{\alpha+\beta} B_{q}(\beta+1, \alpha), \\
& \int_{0}^{\eta} \int_{0}^{s}(\eta-p s)^{\frac{\alpha-1}{p}}(s-q t) \frac{\beta-1}{q} d_{q} t d_{p} s=\frac{\eta^{\alpha+\beta}}{[\beta]_{q}} B_{q}(\beta+1, \alpha) .
\end{aligned}
$$

Lemma 2.4 ([48]) For $\alpha, \beta>0, p, q \in(0,1)$, and $\omega>0$,

$$
\begin{aligned}
& \int_{\omega_{0}}^{t}\left(t-\sigma_{q, \omega}(s)\right) \frac{\alpha-1}{q, \omega}\left(s-\omega_{0}\right)^{\beta} d_{q, \omega} s=\left(t-\omega_{0}\right)^{\alpha+\beta} B_{q}(\beta+1, \alpha), \\
& \int_{\omega_{0}}^{t} \int_{\omega_{0}}^{x}\left(t-\sigma_{p, \omega}(x)\right)_{p, \omega}^{\alpha-1}\left(x-\sigma_{q, \omega}(s)\right) \frac{\beta-1}{q, \omega} d_{q, \omega} s d_{p, \omega} x=\frac{\left(t-\omega_{0}\right)^{\alpha+\beta}}{[\beta]_{q}} B_{q}(\beta+1, \alpha) .
\end{aligned}
$$


Lemma 2.5 Let $\alpha, \beta, \gamma \in(0,1], \alpha+\beta \in(1,2] ; 0<q<1 ; \omega>0 ; T>\omega_{0} ; \lambda_{1}, \lambda_{2}, \mu_{1}, \mu_{2} \in \mathbb{R}^{+}$; $h \in C([0, T], \mathbb{R})$ be a given function; $\phi_{1}, \phi_{2}: C([0, T], \mathbb{R}) \rightarrow \mathbb{R}$ be given functionals. Then the linear variant of problem (1.1) given by

$$
\begin{aligned}
& D_{q, \omega}^{\alpha} D_{q}^{\beta} u(t)=h(t), \quad t \in I_{q, \omega}^{I_{q}^{[0, T]}}, \\
& \lambda_{1} u(\eta)+\lambda_{2} D_{q}^{\gamma} u(\eta)=\phi_{1}(u), \quad \eta \in(0, T), \\
& \mu_{1} u(T)+\mu_{2} D_{q, \omega}^{\gamma} u(T)=\phi_{2}(u)
\end{aligned}
$$

has the unique solution, which is in a form

$$
\begin{aligned}
u(t)= & \frac{1}{\Gamma_{q}(\alpha) \Gamma_{q}(\beta)} \int_{0}^{t} \int_{\omega_{0}}^{x}\left(t-\sigma_{q}(s)\right) \frac{\beta-1}{q}\left(x-\sigma_{q, \omega}(s)\right)_{q, \omega}^{\frac{\alpha-1}{h}} h(s) d_{q, \omega} s d_{q} x \\
& +\left\{\mathbf{A}_{T} \Phi_{\eta}\left[\phi_{1}(u), h\right]-\mathbf{A}_{\eta} \Phi_{T}\left[\phi_{2}(u), h\right]\right\} \frac{1}{\Omega \Gamma_{q}(\beta)} \int_{0}^{t}\left(t-\sigma_{q}(s)\right) \frac{\beta-1}{q}\left(s-\omega_{0}\right)^{\alpha-1} d_{q} s \\
& -\left\{\mathbf{B}_{T} \Phi_{\eta}\left[\phi_{1}(u), h\right]-\mathbf{B}_{\eta} \Phi_{T}\left[\phi_{2}(u), h\right]\right\} \frac{t^{\beta-1}}{\Omega}
\end{aligned}
$$

for $t \in[0, T]$, and the functionals $\Phi_{\eta}\left[\phi_{1}(u), h\right], \Phi_{T}\left[\phi_{2}(u), h\right]$ are defined by

$$
\begin{aligned}
\Phi_{\eta}\left[\phi_{1}(u), h\right]:= & \phi_{1}(u)-\frac{\lambda_{1}}{\Gamma_{q}(\alpha) \Gamma_{q}(\beta)} \int_{0}^{\eta} \int_{\omega_{0}}^{x}\left(\eta-\sigma_{q}(x)\right) \frac{\beta-1}{q} \\
& \times\left(x-\sigma_{q, \omega}(s) \frac{\alpha-1}{q, \omega} h(s) d_{q, \omega} s d_{q} x\right. \\
& -\frac{\lambda_{2}}{\Gamma_{q}(\alpha) \Gamma_{q}(\beta) \Gamma_{q}(-\gamma)} \int_{0}^{\eta} \int_{0}^{r} \int_{\omega_{0}}^{x}\left(\eta-\sigma_{q}(r)\right) \frac{-\gamma-1}{q} \\
& \times\left(r-\sigma_{q}(x)\right) \frac{\beta-1}{q}\left(x-\sigma_{q, \omega}(s)\right)_{q, \omega}^{\frac{\alpha-1}{q}} h(s) d_{q, \omega} s d_{q} x d_{q} r, \\
\Phi_{T}\left[\phi_{2}(u), h\right]:= & \phi_{2}(u)-\frac{\mu_{1}}{\Gamma_{q}(\alpha) \Gamma_{q}(\beta)} \int_{0}^{T} \int_{\omega_{0}}^{x}\left(T-\sigma_{q}(x)\right) \frac{\beta-1}{q} \\
& \times\left(x-\sigma_{q, \omega}(s)\right)_{q, \omega}^{\alpha-1} h(s) d_{q, \omega} s d_{q} x \\
& -\frac{\mu_{2}}{\Gamma_{q}(\alpha) \Gamma_{q}(\beta) \Gamma_{q}(-\gamma)} \int_{\omega_{0}}^{T} \int_{0}^{r} \int_{\omega_{0}}^{x}\left(T-\sigma_{q, \omega}(r)\right) \frac{-\gamma-1}{q, \omega} \\
& \times\left(r-\sigma_{q}(x)\right)_{q} \frac{\beta-1}{q}\left(x-\sigma_{q, \omega}(s)\right)_{q, \omega}^{\alpha-1} h(s) d_{q, \omega} s d_{q} x d_{q, \omega} r,
\end{aligned}
$$

and the constants $\mathbf{A}_{\eta}, \mathbf{A}_{T}, \mathbf{B}_{\eta}, \mathbf{B}_{T}$, and $\Omega$ are defined by

$$
\begin{aligned}
\mathbf{A}_{\eta}:= & \lambda_{1} \eta^{\beta-1}+\frac{\lambda_{2}}{\Gamma_{q}(-\gamma)} \int_{0}^{\eta}\left(\eta-\sigma_{q}(s)\right) \frac{-\gamma-1}{q} s^{\beta-1} d_{q} s, \\
\mathbf{A}_{T}:= & \mu_{1} T^{\beta-1}+\frac{\mu_{2}}{\Gamma_{q}(-\gamma)} \int_{\omega_{0}}^{T}\left(T-\sigma_{q, \omega}(s)\right) \frac{-\gamma-1}{q} s^{\beta-1} d_{q, \omega} s, \\
\mathbf{B}_{\eta}:= & \frac{\lambda_{1}}{\Gamma_{q}(\beta)} \int_{0}^{\eta}\left(\eta-\sigma_{q}(s)\right) \frac{\beta-1}{q}\left(s-\omega_{0}\right)^{\alpha-1} d_{q} s \\
& +\frac{\lambda_{2}}{\Gamma_{q}(\beta) \Gamma_{q}(-\gamma)} \int_{0}^{\eta} \int_{0}^{x}\left(\eta-\sigma_{q}(x)\right) \frac{-\gamma-1}{q}\left(x-\sigma_{q}(s)\right) \frac{\beta-1}{q}\left(s-\omega_{0}\right)^{\alpha-1} d_{q} s d_{q} x,
\end{aligned}
$$




$$
\begin{aligned}
\mathbf{B}_{T}:= & \frac{\mu_{1}}{\Gamma_{q}(\beta)} \int_{0}^{T}\left(T-\sigma_{q}(s)\right) \frac{\beta-1}{q}\left(s-\omega_{0}\right)^{\alpha-1} d_{q} s \\
& +\frac{\mu_{2}}{\Gamma_{q}(\beta) \Gamma_{q}(-\gamma)} \int_{\omega_{0}}^{T} \int_{0}^{x}\left(T-\sigma_{q, \omega}(x)\right) \frac{-\gamma-1}{q, \omega}\left(x-\sigma_{q}(s)\right) \frac{\beta-1}{q}\left(s-\omega_{0}\right)^{\alpha-1} d_{q} s d_{q, \omega} x, \\
\Omega:= & \mathbf{A}_{T} \mathbf{B}_{\eta}-\mathbf{A}_{\eta} \mathbf{B}_{T} \neq 0 .
\end{aligned}
$$

Proof We first take fractional Hahn integral of order $\alpha$ for (2.1). Then the problem becomes fractional $q$-difference equation as follows:

$$
\begin{aligned}
D_{q}^{\beta} u(t) & =C_{0}\left(t-\omega_{0}\right)^{\alpha-1}+\frac{(1-q)\left(t-\omega_{0}\right)^{\alpha}}{\Gamma_{q}(\alpha)} \sum_{k=0}^{\infty} q^{k}\left(1-q^{k+1}\right)_{q}^{\frac{\alpha-1}{q}} h\left(\sigma_{q, \omega}^{k}(t)\right) \\
& =C_{0}\left(t-\omega_{0}\right)^{\alpha-1}+\frac{1}{\Gamma_{q}(\alpha)} \int_{\omega_{0}}^{t}\left(t-\sigma_{q, \omega}(s)\right)_{q, \omega}^{\alpha-1} h(x) d_{q, \omega} s
\end{aligned}
$$

for $t \in I_{q}^{[0, T]}:=\left\{q^{n} s: s \in[0, T], n \in \mathbb{N}_{0}\right\} \cup\{0\}$.

After taking fractional $q$-integral of order $\beta$ for (2.10), we get the solution which is in the form

$$
\begin{aligned}
u(t)= & C_{1} t^{\beta-1}+\frac{C_{0}}{\Gamma_{q}(\beta)}(1-q) t^{\beta} \sum_{k=0}^{\infty} q^{k}\left(1-q^{k+1}\right) \frac{\beta-1}{q}\left(\sigma_{q, \omega}^{k}(t)-\omega_{0}\right)^{\alpha-1} \\
& +\frac{1}{\Gamma_{q}(\alpha) \Gamma_{q}(\beta)}(1-q)^{2}\left(t-\omega_{0}\right)^{\beta} \sum_{h=0}^{\infty} \sum_{k=0}^{\infty} q^{h+k}\left(1-q^{h+1}\right) \frac{\beta-1}{q} \\
& \times\left(1-q^{k+1}\right)_{q}^{\frac{\alpha-1}{q}}\left(\sigma_{q, \omega}^{h}(t)\right)^{\alpha} h\left(\sigma_{q, \omega}^{k}\left(\sigma_{q}^{h}(t)\right)\right) \\
= & C_{1} t^{\beta-1}+\frac{C_{0}}{\Gamma_{q}(\beta)} \int_{0}^{t}\left(t-\sigma_{q}(s)\right) \frac{\beta-1}{q}\left(s-\omega_{0}\right)^{\alpha-1} d_{q} s \\
& +\frac{1}{\Gamma_{q}(\alpha) \Gamma_{q}(\beta)} \int_{0}^{t} \int_{\omega_{0}}^{x}\left(t-\sigma_{q}(x)\right) \frac{\beta-1}{q}\left(x-\sigma_{q, \omega}(s)\right)_{q, \omega}^{\frac{\alpha-1}{q}} h(s) d_{q, \omega} s d_{q} x
\end{aligned}
$$

for $t \in[0, T]$.

In order to find the unknown constants $C_{1}$ and $C_{0}$ that appeared in (2.11), we first take fractional $q$-difference and fractional Hahn difference of order $\gamma$ for (2.11) to get

$$
\begin{aligned}
D_{q}^{\gamma} u(t)= & \frac{C_{1}}{\Gamma_{q}(-\gamma)} \int_{0}^{t}\left(t-\sigma_{q}(s)\right) \frac{-\gamma-1}{q} s^{\beta-1} d_{q} s \\
& +\frac{C_{0}}{\Gamma_{q}(\beta) \Gamma_{q}(-\gamma)} \int_{0}^{t} \int_{0}^{x}\left(t-\sigma_{q}(x)\right) \frac{-\gamma-1}{q}\left(x-\sigma_{q}(s)\right) \frac{\beta-1}{q}\left(s-\omega_{0}\right)^{\alpha-1} d_{q} s d_{q} x \\
& +\frac{1}{\Gamma_{q}(\alpha) \Gamma_{q}(\beta) \Gamma_{q}(-\gamma)} \int_{0}^{t} \int_{0}^{r} \int_{\omega_{0}}^{x}\left(t-\sigma_{q}(r)\right) \frac{-\gamma-1}{q}\left(r-\sigma_{q}(x)\right) \frac{\beta-1}{q} \\
& \times\left(x-\sigma_{q, \omega}(s)\right) \frac{\alpha-1}{q, \omega} h(s) d_{q} s d_{q} x d_{q, \omega} r
\end{aligned}
$$


for $t \in[0, T]$, and

$$
\begin{aligned}
D_{q, \omega}^{\gamma} u(t)= & \frac{C_{1}}{\Gamma_{q}(-\gamma)} \int_{\omega_{0}}^{t}\left(t-\sigma_{q, \omega}(s)\right) \frac{-\gamma-1}{q, \omega} s^{\beta-1} d_{q, \omega} s \\
& +\frac{C_{0}}{\Gamma_{q}(\beta) \Gamma_{q}(-\gamma)} \int_{\omega_{0}}^{t} \int_{0}^{x}\left(t-\sigma_{q, \omega}(x)\right) \frac{-\gamma-1}{q, \omega}\left(x-\sigma_{q}(s)\right) \frac{\beta-1}{q}\left(s-\omega_{0}\right)^{\alpha-1} d_{q} s d_{q, \omega} x \\
& +\frac{1}{\Gamma_{q}(\alpha) \Gamma_{q}(\beta) \Gamma_{q}(-\gamma)} \int_{\omega_{0}}^{t} \int_{0}^{r} \int_{\omega_{0}}^{x}\left(t-\sigma_{q, \omega}(r)\right) \frac{-\gamma-1}{q, \omega}\left(r-\sigma_{q}(x)\right) \frac{\beta-1}{q} \\
& \times\left(x-\sigma_{q, \omega}(s)\right) \frac{\alpha-1}{q, \omega} h(s) d_{q, \omega} s d_{q} x d_{q, \omega} r
\end{aligned}
$$

for $t \in\left[\omega_{0}, T\right]$, respectively.

Substitute $t=\eta$ into (2.11) and (2.12) and use the first condition of (2.1). Then we get

$$
\mathbf{A}_{\eta} C_{1}+\mathbf{B}_{\eta} C_{0}=\Phi_{\eta}\left[\phi_{1}, h\right]
$$

Similarly, substitute $t=T$ into (2.11) and (2.13) and employ the second condition of (2.1). We have

$$
\mathbf{A}_{T} C_{1}+\mathbf{B}_{T} C_{0}=\Phi_{T}\left[\phi_{2}, h\right]
$$

We can solve the linear system for (2.14)-(2.15), and we find that

$$
C_{1}=\frac{\mathbf{B}_{\eta} \Phi_{T}\left[\phi_{2}(u), h\right]-\mathbf{B}_{T} \Phi_{\eta}\left[\phi_{1}(u), h\right]}{\Omega}
$$

and

$$
C_{0}=\frac{\mathbf{A}_{T} \Phi_{\eta}\left[\phi_{1}(u), h\right]-\mathbf{A}_{\eta} \Phi_{T}\left[\phi_{2}(u), h\right]}{\Omega},
$$

where $\Phi_{\eta}\left[\phi_{1}(u), h\right], \Phi_{T}\left[\phi_{2}(u), h\right], \mathbf{A}_{\eta}, \mathbf{A}_{T}, \mathbf{B}_{\eta}, \mathbf{B}_{T}, \Omega$ are defined by (2.3)-(2.9), respectively. Solution (2.2) can be exposed after substituting $C_{1}$ and $C_{0}$ into (2.11).

\section{Existence and uniqueness result}

In this section, we employ the Banach fixed point theorems to consider the existence and uniqueness result for problem (1.1). Let $\mathcal{C}=C([0, T], \mathbb{R})$ be a Banach space of all function $u$ with the norm defined by

$$
\|u\|_{\mathcal{C}}=\|u\|+\left\|D_{q}^{\theta} u\right\|+\left\|D_{q, \omega}^{v} u\right\|
$$

where

$$
\|u\|=\max _{t \in[0, T]}|u(t)|, \quad\left\|D_{q}^{\theta} u\right\|=\max _{t \in[0, T]}\left|D_{q}^{\theta} u(t)\right|,
$$

and

$$
\left\|D_{q, \omega}^{v} u\right\|=\max _{t \in\left[\omega_{0}, T\right]}\left|D_{q, \omega}^{v} u(t)\right| .
$$


We define an operator $\mathcal{F}: \mathcal{C} \rightarrow \mathcal{C}$ by

$$
\begin{aligned}
(\mathcal{F} u)(t):= & \frac{1}{\Gamma_{q}(\alpha) \Gamma_{q}(\beta)} \int_{0}^{t} \int_{\omega_{0}}^{x}\left(t-\sigma_{q}(x)\right) \frac{\beta-1}{q}\left(x-\sigma_{q, \omega}(s)\right)_{q, \omega}^{\frac{\alpha-1}{}} \\
& \times F\left[s, u(s), D_{q}^{\theta} u(s), D_{q, \omega}^{\nu} u(s)\right] d_{q, \omega} s d_{q} x \\
& +\left\{\mathbf{A}_{T}^{*} \Phi_{\eta}\left[\phi_{1}(u), F_{u}\right]-\mathbf{A}_{\eta}^{*} \Phi_{T}\left[\phi_{2}(u), F_{u}\right]\right\} \frac{1}{\Omega \Gamma_{q}(\beta)} \\
& \times \int_{0}^{t}\left(t-\sigma_{q}(s)\right)_{q}^{\frac{\beta-1}{q}}\left(s-\omega_{0}\right)^{\alpha-1} d_{q} s \\
& -\left\{\mathbf{B}_{T}^{*} \Phi_{\eta}\left[\phi_{1}(u), F_{u}\right]-\mathbf{B}_{\eta}^{*} \Phi_{T}\left[\phi_{2}(u), F_{u}\right]\right\} \frac{t^{\beta-1}}{\Omega}
\end{aligned}
$$

where

$$
\begin{aligned}
\Phi_{\eta}^{*}\left[\phi_{1}(u), F_{u}\right]:= & \phi_{1}(u)-\frac{\lambda_{1}}{\Gamma_{q}(\alpha) \Gamma_{q}(\beta)} \int_{0}^{\eta} \int_{\omega_{0}}^{x}\left(\eta-\sigma_{q}(x)\right) \frac{\beta-1}{q}\left(x-\sigma_{q, \omega}(s)\right)_{q, \omega}^{\frac{\alpha-1}{}} \\
& \times F\left[s, u(s), D_{q}^{\theta} u(s), D_{q, \omega}^{v} u(s)\right] d_{q, \omega} s d_{q} x-\frac{\lambda_{2}}{\Gamma_{q}(\alpha) \Gamma_{q}(\beta) \Gamma_{q}(-\gamma)} \\
& \times \int_{0}^{\eta} \int_{0}^{r} \int_{\omega_{0}}^{x}\left(\eta-\sigma_{q}(r)\right) \frac{\gamma-1}{q}\left(r-\sigma_{q}(x)\right) \frac{\beta-1}{q}\left(x-\sigma_{q, \omega}(s)\right)_{q, \omega}^{\frac{\alpha-1}{}} \\
& \times F\left[s, u(s), D_{q}^{\theta} u(s), D_{q, \omega}^{v} u(s)\right] d_{q, \omega} s d_{q} x d_{q} r, \\
\Phi_{T}^{*}\left[\phi_{2}(u), F_{u}\right]:= & \phi_{2}(u)-\frac{\mu_{1}}{\Gamma_{q}(\alpha) \Gamma_{q}(\beta)} \int_{0}^{T} \int_{\omega_{0}}^{x}\left(T-\sigma_{q}(x)\right) \frac{\beta-1}{q}\left(x-\sigma_{q, \omega}(s)\right) \frac{\alpha-1}{q, \omega} \\
& \times F\left[s, u(s), D_{q}^{\theta} u(s), D_{q, \omega}^{v} u(s)\right] d_{q, \omega} s d_{q} x-\frac{\mu_{2}}{\Gamma_{q}(\alpha) \Gamma_{q}(\beta) \Gamma_{q}(-\gamma)} \\
& \times \int_{0}^{T} \int_{0}^{r} \int_{\omega_{0}}^{x}\left(T-\sigma_{q}(r)\right)_{q} \frac{\gamma-1}{q}\left(r-\sigma_{q}(x)\right) \frac{\beta-1}{q}\left(x-\sigma_{q, \omega}(s)\right) \frac{\alpha-1}{q, \omega} \\
& \times F\left[s, u(s), D_{q}^{\theta} u(s), D_{q, \omega}^{v} u(s)\right] d_{q, \omega} s d_{q} x d_{q} r,
\end{aligned}
$$

and the constants $\mathbf{A}_{\eta}, \mathbf{A}_{T}, \mathbf{B}_{\eta}, \mathbf{B}_{T}, \Omega$ are defined by (2.5)-(2.9), respectively.

If one can prove that $\mathcal{F}$ has a fixed point, we can conclude that problem (1.1) has a solution.

Theorem 3.1 Let $F:[0, T] \times \mathbb{R} \times \mathbb{R} \times \mathbb{R} \rightarrow \mathbb{R}$ be a continuous function, and assume that the following conditions hold:

$\left(H_{1}\right)$ There exist constants $\ell_{1}, \ell_{2}, \ell_{3}>0$ such that, for each $t \in[0, T]$ and $u_{i}, v_{i} \in \mathbb{R}, i=$ $1,2,3$,

$$
\left|F\left[t, u_{1}, u_{2}, u_{3}\right]-F\left[t, v_{1}, v_{2}, v_{3}\right]\right| \leq \ell_{1}\left|u_{1}-v_{1}\right|+\ell_{2}\left|u_{2}-v_{2}\right|+\ell_{3}\left|u_{3}-v_{3}\right| .
$$

$\left(H_{2}\right)$ There exist constants $\xi_{1}, \xi_{2}>0$ such that, for each $u, v \in \mathcal{C}$,

$$
\left|\phi_{1}(u)-\phi_{1}(v)\right| \leq \xi_{1}\|u-v\|_{\mathcal{C}} \text { and }\left|\phi_{2}(u)-\phi_{2}(v)\right| \leq \xi_{2}\|u-v\|_{\mathcal{C}} .
$$

$\left(H_{3}\right) \mathcal{X}:=\left(\ell_{1}+\ell_{2}+\ell_{3}\right) \Theta+\xi_{1} \Upsilon_{T}+\xi_{2} \Upsilon_{\eta}<\frac{1}{3}$, 
where

$$
\begin{aligned}
& \Theta:=\frac{\left(T-\omega_{0}\right)^{\alpha} T^{\beta}}{\Gamma_{q}(\alpha+1) \Gamma_{q}(\beta+1)}+\mathcal{O}_{1} \Upsilon_{T}+\mathcal{O}_{2} \Upsilon_{\eta}, \\
& \mathcal{O}_{1}:=\frac{\left(\eta-\omega_{0}\right)^{\alpha} \eta^{\beta}}{\Gamma_{q}(\alpha+1) \Gamma_{q}(\beta+1)}\left|\lambda_{1}-\frac{\lambda_{2} \eta^{-\gamma}}{\Gamma_{q}(1-\gamma)}\right|, \\
& \mathcal{O}_{2}:=\frac{\left(T-\omega_{0}\right)^{\alpha} T^{\beta}}{\Gamma_{q}(\alpha+1) \Gamma_{q}(\beta+1)}\left|\mu_{1}-\frac{\mu_{2}\left(T-\omega_{0}\right)^{-\gamma}}{\Gamma_{q}(1-\gamma)}\right|, \\
& \Upsilon_{T}:=\frac{1}{|\Omega|}\left\{\left|\mathbf{A}_{T}\right| \frac{\left(T-\omega_{0}\right)^{\alpha-1} T^{\beta}}{\Gamma_{q}(\beta+1)}+\left|\mathbf{B}_{T}\right| T^{\beta-1}\right\} \\
& \Upsilon_{\eta}:=\frac{1}{|\Omega|}\left\{\left|\mathbf{A}_{\eta}\right| \frac{\left(T-\omega_{0}\right)^{\alpha-1} T^{\beta}}{\Gamma_{q}(\beta+1)}+\left|\mathbf{B}_{\eta}\right| T^{\beta-1}\right\} .
\end{aligned}
$$

Then problem (1.1) has a unique solution.

Proof Let $\mathcal{H}|u-v|(t):=\left|F\left[t, u, D_{q}^{\theta} u, D_{q, \omega}^{v} u\right]-F\left[t, v, D_{q}^{\theta} v, D_{q, \omega}^{v} v\right]\right|$. For each $t \in[0, T]$ and $u, v \in \mathcal{C}$, from (3.2), we see that

$$
\begin{aligned}
\mid \Phi_{\eta}^{*} & {\left[\phi_{1}(u), F_{u}\right]-\Phi_{\eta}^{*}\left[\phi_{1}(u), F_{v}\right] \mid } \\
\leq & \left|\phi_{1}(u)-\phi_{1}(v)\right|+\mid \frac{\lambda_{1}}{\Gamma_{q}(\alpha) \Gamma_{q}(\beta)} \int_{0}^{\eta} \int_{\omega_{0}}^{x}\left(\eta-\sigma_{q}(x)\right)_{q}^{\frac{\beta-1}{q}}\left(x-\sigma_{q, \omega}(s)\right)_{q, \omega}^{\frac{\alpha-1}{}} \\
& \times \mathcal{H}|u-v|(s) d_{q, \omega} s d_{q} x-\frac{\lambda_{2}}{\Gamma_{q}(\alpha) \Gamma_{q}(\beta) \Gamma_{q}(-\gamma)} \int_{0}^{\eta} \int_{0}^{r} \int_{\omega_{0}}^{x}\left(\eta-\sigma_{q}(r)\right)_{q} \frac{\gamma-1}{q} \\
& \times\left(r-\sigma_{q}(x)\right)_{q} \frac{\beta-1}{q}\left(x-\sigma_{q, \omega}(s) \frac{\alpha-1}{q, \omega} \mathcal{H}|u-v|(s) d_{q, \omega} s d_{q} x d_{q} r \mid\right. \\
\leq & \xi_{1}\|u-v\|_{\mathcal{C}}+\left(\ell_{1}|u-v|+\ell_{2}\left|D_{q}^{\theta} u-D_{q}^{\theta} v\right|+\ell_{3}\left|D_{q, \omega}^{v} u-D_{q, \omega}^{v} v\right|\right) \\
& \times\left|\frac{\lambda_{1}\left(\eta-\omega_{0}\right)^{\alpha} \eta^{\beta}}{\Gamma_{q}(\alpha+1) \Gamma_{q}(\beta+1)}-\frac{\lambda_{2}\left(\eta-\omega_{0}\right)^{\alpha} \eta^{\beta-\gamma}}{\Gamma_{q}(\alpha+1) \Gamma_{q}(\beta+1) \Gamma_{q}(1-\gamma)}\right| \\
\leq & \xi_{1}\|u-v\|_{\mathcal{C}}+\left(\ell_{1}|u-v|+\ell_{2}\left|D_{q}^{\theta} u-D_{q}^{\theta} v\right|+\ell_{3}\left|D_{q, \omega}^{v} u-D_{q, \omega}^{v} v\right|\right) \mathcal{O}_{1} \\
\leq & {\left[\xi_{1}+\left(\ell_{1}+\ell_{2}+\ell_{3}\right) \mathcal{O}_{1}\right]\|u-v\|_{\mathcal{C} .} }
\end{aligned}
$$

Similarly, we see from (3.3) that

$$
\left|\Phi_{T}^{*}\left[\phi_{2}(u), F_{u}\right]-\Phi_{T}^{*}\left[\phi_{2}(u), F_{v}\right]\right| \leq\left[\xi_{2}+\left(\ell_{1}+\ell_{2}+\ell_{3}\right) \mathcal{O}_{2}\right]\|u-v\|_{\mathcal{C}}
$$

Consider

$$
\begin{aligned}
|(\mathcal{F} u)(t)-(\mathcal{F} v)(t)| & \\
\leq & \frac{1}{\Gamma_{q}(\alpha) \Gamma_{q}(\beta)} \int_{0}^{T} \int_{\omega_{0}}^{x}\left(T-\sigma_{q}(x)\right) \frac{\beta-1}{q}\left(x-\sigma_{q, \omega}(s)\right) \frac{\alpha-1}{q, \omega} \mathcal{H}|u-v|(s) d_{q, \omega} s d_{q} x \\
& +\left\{\left|\mathbf{A}_{T}\right|\left|\Phi_{\eta}^{*}\left[\phi_{1}(u), F_{u}\right]-\Phi_{\eta}^{*}\left[\phi_{1}(u), F_{v}\right]\right|+\left|\mathbf{A}_{\eta}\right|\left|\Phi_{T}^{*}\left[\phi_{2}(u), F_{u}\right]-\Phi_{T}^{*}\left[\phi_{2}(u), F_{v}\right]\right|\right\} \\
& \times \frac{\left(T-\omega_{0}\right)^{\alpha-1} T^{\beta}}{|\Omega| \Gamma_{q}(\beta+1)}
\end{aligned}
$$




$$
\begin{aligned}
& +\left\{\left|\mathbf{B}_{T}\right|\left|\Phi_{\eta}^{*}\left[\phi_{1}(u), F_{u}\right]-\Phi_{\eta}^{*}\left[\phi_{1}(u), F_{v}\right]\right|+\left|\mathbf{B}_{\eta}\right|\left|\Phi_{T}^{*}\left[\phi_{2}(u), F_{u}\right]-\Phi_{T}^{*}\left[\phi_{2}(u), F_{v}\right]\right|\right\} \\
& \times \frac{T^{\beta-1}}{\Omega} \\
\leq & \|u-v\|_{\mathcal{C}}\left[\frac{\left(\ell_{1}+\ell_{2}+\ell_{3}\right)\left(T-\omega_{0}\right)^{\alpha} T^{\beta}}{\Gamma_{q}(\alpha+1) \Gamma_{q}(\beta+1)}+\frac{\left[\xi_{1}+\left(\ell_{1}+\ell_{2}+\ell_{3}\right) \mathcal{O}_{1}\right]}{|\Omega|}\right. \\
& \times\left\{\left|\mathbf{A}_{T}\right| \frac{\left(T-\omega_{0}\right)^{\alpha-1} T^{\beta}}{\Gamma_{q}(\beta+1)}+\left|\mathbf{B}_{T}\right| T^{\beta-1}\right\} \\
& \left.+\frac{\left[\xi_{2}+\left(\ell_{1}+\ell_{2}+\ell_{3}\right) \mathcal{O}_{2}\right]}{|\Omega|}\left\{\left|\mathbf{A}_{\eta}\right| \frac{\left(T-\omega_{0}\right)^{\alpha-1} T^{\beta}}{\Gamma_{q}(\beta+1)}+\left|\mathbf{B}_{\eta}\right| T^{\beta-1}\right\}\right] \\
\leq & {\left[\left(\ell_{1}+\ell_{2}+\ell_{3}\right) \Theta+\xi_{1} \Upsilon_{T}+\xi_{2} \Upsilon_{\eta}\right]\|u-v\|_{\mathcal{C}} } \\
= & \mathcal{X}\|u-v\|_{\mathcal{C}} .
\end{aligned}
$$

Taking fractional fractional $q$-difference of order $\theta$ and fractional Hahn difference of order $v$ for (3.1), we get

$$
\begin{aligned}
D_{q}^{\theta} u(t)= & \frac{1}{\Gamma_{q}(\alpha) \Gamma_{q}(\beta) \Gamma_{p}(-\theta)} \int_{0}^{t} \int_{0}^{r} \int_{\omega_{0}}^{x}\left(t-\sigma_{q}(r)\right)_{q}^{\frac{-\theta-1}{q}}\left(r-\sigma_{q}(x)\right)_{q}^{\frac{\beta-1}{q}} \\
& \times\left(x-\sigma_{q, \omega}(s)\right)_{q, \omega}^{\alpha-1} F\left[s, u(s), D_{q}^{\theta} u(s), D_{q, \omega}^{\phi} u(s)\right] d_{q} s d_{q} x d_{q, \omega} r \\
& +\frac{\left\{\mathbf{A}_{T}^{*} \Phi_{\eta}\left[\phi_{1}(u), F_{u}\right]-\mathbf{A}_{\eta}^{*} \Phi_{T}\left[\phi_{2}(u), F_{u}\right]\right\}}{\Omega \Gamma_{q}(\beta) \Gamma_{p}(-\theta)} \int_{0}^{t} \int_{0}^{x}\left(t-\sigma_{q}(x)\right)_{q}^{\frac{-\theta-1}{q}} \\
& \times\left(x-\sigma_{q}(s) \frac{\beta-1}{q}\left(s-\omega_{0}\right)^{\alpha-1} d_{q} s d_{q} x,\right. \\
& -\frac{\left\{\mathbf{B}_{T}^{*} \Phi_{\eta}\left[\phi_{1}(u), F_{u}\right]-\mathbf{B}_{\eta}^{*} \Phi_{T}\left[\phi_{2}(u), F_{u}\right]\right\}}{\Omega \Gamma_{q}(-\theta)} \int_{0}^{t}\left(t-\sigma_{q}(s)\right)_{q}^{-\theta-1} s^{\beta-1} d_{q} s
\end{aligned}
$$

for $t \in[0, T]$, and

$$
\begin{aligned}
D_{q, \omega}^{v} u(t)= & \frac{1}{\Gamma_{q}(\alpha) \Gamma_{q}(\beta) \Gamma_{p}(-v)} \int_{\omega_{0}}^{t} \int_{0}^{r} \int_{\omega_{0}}^{x}\left(t-\sigma_{q, \omega}(r)\right) \frac{-v-1}{q, \omega}\left(r-\sigma_{q}(x)\right) \frac{\beta-1}{q} \\
& \times\left(x-\sigma_{q, \omega}(s)\right) \frac{\alpha-1}{q, \omega} F\left[s, u(s), D_{q}^{\theta} u(s), D_{q, \omega}^{v} u(s)\right] d_{q, \omega} s d_{q} x d_{q, \omega} r \\
& +\frac{\left\{\mathbf{A}_{T}^{*} \Phi_{\eta}\left[\phi_{1}(u), F_{u}\right]-\mathbf{A}_{\eta}^{*} \Phi_{T}\left[\phi_{2}(u), F_{u}\right]\right\}}{\Omega \Gamma_{q}(\beta) \Gamma_{p}(-v)} \int_{\omega_{0}}^{t} \int_{0}^{x}\left(t-\sigma_{q, \omega}(x)\right) \frac{-v-1}{q, \omega} \\
& \times\left(x-\sigma_{q}(s)\right) \frac{\beta-1}{q}\left(s-\omega_{0}\right)^{\alpha-1} d_{q} s d_{q, \omega} x, \\
& -\frac{\left\{\mathbf{B}_{T}^{*} \Phi_{\eta}\left[\phi_{1}(u), F_{u}\right]-\mathbf{B}_{\eta}^{*} \Phi_{T}\left[\phi_{2}(u), F_{u}\right]\right\}}{\Omega \Gamma_{q}(-v)} \int_{\omega_{0}}^{t}\left(t-\sigma_{q, \omega}(s)\right)_{q, \omega}^{-v-1} s^{\beta-1} d_{q, \omega} s
\end{aligned}
$$

for $t \in\left[\omega_{0}, T\right]$, respectively.

Similarly, we have

$$
\begin{aligned}
& \left|\left(D_{q}^{\theta} \mathcal{F} u\right)(t)-\left(D_{q}^{\theta} \mathcal{F} v\right)(t)\right|<\mathcal{X}\|u-v\|_{\mathcal{C}}, \\
& \left|\left(D_{q, \omega}^{v} \mathcal{F} u\right)(t)-\left(D_{q, \omega}^{v} \mathcal{F} v\right)(t)\right|<\mathcal{X}\|u-v\|_{\mathcal{C}} .
\end{aligned}
$$


From (3.9), (3.12), and (3.13), it implies that

$$
\begin{aligned}
\|\mathcal{F} u-\mathcal{F} v\|_{\mathcal{C}} & =\|\mathcal{F} u-\mathcal{F} v\|+\left\|D_{q}^{\theta} \mathcal{F} u-D_{q}^{\theta} \mathcal{F} v\right\|+\left\|D_{q, \omega}^{v} \mathcal{F} u-D_{q, \omega}^{v} \mathcal{F} v\right\| \\
& <3 \mathcal{X}\|u-v\|_{\mathcal{C}} .
\end{aligned}
$$

Therefore, $\mathcal{F}$ is a contraction by $\left(H_{3}\right)$. Thus, $\mathcal{F}$ has a fixed point, which is a unique solution of problem (1.1) by using the Banach fixed point theorem.

\section{Existence of at least one solution}

In this section, we also prove the existence of at least one solution of (1.1) by using Schauder's fixed point theorem. Firstly, we provide some basic knowledge that is used in this section as follows.

Lemma 4.1 ([59] (Arzelá-Ascoli theorem)) A set of functions in $C[a, b]$ with the sup norm is relatively compact if and only if it is uniformly bounded and equicontinuous on $[a, b]$.

Lemma 4.2 ([59]) If a set is closed and relatively compact, then it is compact.

Lemma 4.3 ([60] (Schauder's fixed point theorem)) Let $(D, d)$ be a complete metric space, $U$ be a closed convex subset of $D$, and $T: D \rightarrow D$ be the map such that the set $T u: u \in U$ is relatively compact in $D$. Then the operator $T$ has at least one fixed point $u^{*} \in U: T u^{*}=u^{*}$.

Based on the above lemmas, we prove the existence of at least one solution of (1.1) as shown in the following theorem.

Theorem 4.1 Suppose that $\left(H_{1}\right)$ and $\left(H_{3}\right)$ hold. Then problem (1.1) has at least one solution.

Proof The proof is divided into three steps as follows.

Step I. We verify that $\mathcal{F}$ maps bounded sets into bounded sets in $B_{R}=\left\{u \in \mathcal{C}:\|u\|_{\mathcal{C}} \leq R\right\}$. We let $\max _{t \in[0, T]}|F(t, 0,0,0)|=M, \sup _{u \in \mathcal{C}}\left|\phi_{1}(u)\right|=N_{1}, \sup _{u \in \mathcal{C}}\left|\phi_{2}(u)\right|=N_{2}$ and choose a constant

$$
R \geq \frac{M \Theta+N_{1} \Upsilon_{T}+N_{2} \Upsilon_{\eta}}{\frac{1}{3}-\left(\ell_{1}+\ell_{2}+\ell_{3}\right) \Theta} .
$$

We denote that

$$
|\mathcal{S}(t, u, 0)|=\left|F\left[t, u, D_{q}^{\theta} u, D_{q, \omega}^{v} u\right]-F[t, 0,0,0]\right|+|F[t, 0,0,0]| .
$$

For each $t \in[0, T]$ and $u \in B_{R}$, we have

$$
\begin{aligned}
\mid \Phi_{\eta}^{*}[ & \left.\phi_{1}(u), F_{u}\right] \mid \\
\leq & N_{1}+\left|\frac{\lambda_{1}}{\Gamma_{q}(\alpha) \Gamma_{q}(\beta)} \int_{0}^{\eta} \int_{\omega_{0}}^{x}\left(\eta-\sigma_{q}(x)\right) \frac{\beta-1}{q}\left(x-\sigma_{q, \omega}(s)\right) \frac{\alpha-1}{q, \omega}\right| \mathcal{S}(s, u, 0) \mid d_{q, \omega} s d_{q} x \\
& \quad-\frac{\lambda_{2}}{\Gamma_{q}(\alpha) \Gamma_{q}(\beta) \Gamma_{q}(-\gamma)} \int_{0}^{\eta} \int_{0}^{r} \int_{\omega_{0}}^{x}\left(\eta-\sigma_{q}(r) \frac{\gamma-1}{q}\left(r-\sigma_{q}(x)\right) \frac{\beta-1}{q}\left(x-\sigma_{q, \omega}(s)\right) \frac{\alpha-1}{q, \omega}\right.
\end{aligned}
$$




$$
\begin{aligned}
& \times|\mathcal{S}(s, u, 0)| d_{q, \omega} s d_{q} x d_{q} r \mid \\
\leq & N_{1}+\left[\left(\ell_{1}+\ell_{2}+\ell_{3}\right)\|u\|_{\mathcal{C}}+M\right] \mathcal{O}_{1} \\
\leq & N_{1}+\left[\left(\ell_{1}+\ell_{2}+\ell_{3}\right) R+M\right] \mathcal{O}_{1} .
\end{aligned}
$$

Similarly, we find that

$$
\left|\Phi_{T}^{*}\left[\phi_{2}(u), F_{u}\right]\right| \leq N_{2}+\left[\left(\ell_{1}+\ell_{2}+\ell_{3}\right) R+M\right] \mathcal{O}_{2} .
$$

From (4.2)-(4.3), we find that

$$
|(\mathcal{F} u)(t)| \leq \Theta\left[\left(\ell_{1}+\ell_{2}+\ell_{3}\right) R+M\right]+N_{1} \Upsilon_{T}+N_{2} \Upsilon_{\eta} \leq \frac{R}{3}
$$

and

$$
\left|\left(D_{q}^{\theta} \mathcal{F} u\right)(t)\right|<\frac{R}{3} \quad \text { and } \quad\left|\left(D_{q, \omega}^{v} \mathcal{F} u\right)(t)\right|<\frac{R}{3} .
$$

Hence,

$$
\|\mathcal{F} u\|_{\mathcal{C}}=\|\mathcal{F} u\|+\left\|D_{q}^{\theta} \mathcal{F} u\right\|+\left\|D_{q, \omega}^{v} \mathcal{F} u\right\|<\frac{R}{3}+\frac{R}{3}+\frac{R}{3}=R,
$$

which implies that $\mathcal{F}$ is uniformly bounded.

Step II. It is obvious that the operator $\mathcal{F}$ is continuous on $B_{R}$ due to the continuity of $F$. Step III. We prove that $\mathcal{F}$ is equicontinuous on $B_{R}$. For any $t_{1}, t_{2} \in I_{q, \omega}^{T}$ with $t_{1}<t_{2}$, we find that

$$
\begin{aligned}
\left|(\mathcal{F} u)\left(t_{2}\right)-(\mathcal{F} u)\left(t_{1}\right)\right| \leq & \frac{\|F\|\left(T-\omega_{0}\right)^{\alpha}}{\Gamma_{q}(\alpha+1) \Gamma_{q}(\beta+1)}\left|t_{2}^{\beta}-t_{1}^{\beta}\right| \\
& \quad+\frac{\left(T-\omega_{0}\right)^{\alpha-1}\left|t_{2}^{\beta}-t_{1}^{\beta}\right|}{|\Omega| \Gamma_{q}(\beta+1)}\left\{\left|\mathbf{A}_{T}\right|\left|\Phi_{\eta}^{*}\left[\phi_{1}, F\right]\right|+\left|\mathbf{A}_{\eta}\right|\left|\Phi_{T}^{*}\left[\phi_{2}, F\right]\right|\right\} \\
& \quad \frac{\left|t_{2}^{\beta-1}-t_{1}^{\beta-1}\right|}{|\Omega|}\left\{\left|\mathbf{B}_{T}\right|\left|\Phi_{\eta}^{*}\left[\phi_{1}, F\right]\right|+\left|\mathbf{B}_{\eta}\right|\left|\Phi_{T}^{*}\left[\phi_{2}, F\right]\right|\right\} \\
\left|\left(D_{q}^{\theta} \mathcal{F} u\right)\left(t_{2}\right)-\left(D_{q}^{\theta} \mathcal{F} u\right)\left(t_{1}\right)\right| & \\
\leq & \frac{\|F\|\left(T-\omega_{0}\right)^{\alpha} T^{\beta}}{\Gamma_{q}(\alpha+1) \Gamma_{q}(\beta+1) \Gamma_{q}(1-\theta)}\left|t_{2}^{-\theta}-t_{1}^{-\theta}\right| \\
+ & \frac{\left(T-\omega_{0}\right)^{\alpha-1} T^{\beta}\left|t_{2}^{-\theta}-t_{1}^{-\theta}\right|}{|\Omega| \Gamma_{q}(\beta+1) \Gamma_{q}(1-\theta)}\left\{\left|\mathbf{A}_{T}\right|\left|\Phi_{\eta}^{*}\left[\phi_{1}, F\right]\right|+\left|\mathbf{A}_{\eta}\right|\left|\Phi_{T}^{*}\left[\phi_{2}, F\right]\right|\right\} \\
+ & \frac{T^{\beta-1}\left|t_{2}^{-\theta}-t_{1}^{-\theta}\right|}{|\Omega| \Gamma_{q}(1-\theta)}\left\{\left|\mathbf{B}_{T}\right|\left|\Phi_{\eta}^{*}\left[\phi_{1}, F\right]\right|+\left|\mathbf{B}_{\eta}\right|\left|\Phi_{T}^{*}\left[\phi_{2}, F\right]\right|\right\}
\end{aligned}
$$

and

$$
\begin{aligned}
& \left|\left(D_{q, \omega}^{v} \mathcal{F} u\right)\left(t_{2}\right)-\left(D_{q, \omega}^{v} \mathcal{F} u\right)\left(t_{1}\right)\right| \\
& \quad \leq \frac{\|F\|\left(T-\omega_{0}\right)^{\alpha} T^{\beta}}{\Gamma_{q}(\alpha+1) \Gamma_{q}(\beta+1) \Gamma_{q}(1-v)}\left|\left(t_{2}-\omega_{0}\right)^{-v}-\left(t_{1}-\omega_{0}\right)^{-v}\right|
\end{aligned}
$$




$$
\begin{aligned}
& +\frac{\left(T-\omega_{0}\right)^{\alpha-1} T^{\beta}\left|\left(t_{2}-\omega_{0}\right)^{-\nu}-\left(t_{1}-\omega_{0}\right)^{-v}\right|}{|\Omega| \Gamma_{q}(\beta+1) \Gamma_{q}(1-v)}\left\{\left|\mathbf{A}_{T}\right|\left|\Phi_{\eta}^{*}\left[\phi_{1}, F\right]\right|+\left|\mathbf{A}_{\eta}\right|\left|\Phi_{T}^{*}\left[\phi_{2}, F\right]\right|\right\} \\
& +\frac{T^{\beta-1}\left|\left(t_{2}-\omega_{0}\right)^{-\nu}-\left(t_{1}-\omega_{0}\right)^{-v}\right|}{|\Omega| \Gamma_{q}(1-v)}\left\{\left|\mathbf{B}_{T}\right|\left|\Phi_{\eta}^{*}\left[\phi_{1}, F\right]\right|+\left|\mathbf{B}_{\eta}\right|\left|\Phi_{T}^{*}\left[\phi_{2}, F\right]\right|\right\}
\end{aligned}
$$

When $\left|t_{2}-t_{1}\right| \rightarrow 0$, we find that the right-hand side of (4.6)-(4.8) tends to be zero. Hence, $\mathcal{F}$ is relatively compact on $B_{R}$ and the set $\mathcal{F}\left(B_{R}\right)$ is an equicontinuous set. From Steps I to III and the Arzelá-Ascoli theorem, we can conclude that $\mathcal{F}: \mathcal{C} \rightarrow \mathcal{C}$ is completely continuous. Therefore, problem (1.1) has at least one solution by Schauder's fixed point theorem.

\section{Example}

Consider the nonlocal Robin boundary value problems for sequential fractional Hahn-qdifference equation as given by

$$
\begin{aligned}
& D_{\frac{1}{2}, \frac{1}{3}}^{\frac{1}{3}} D_{\frac{1}{2}}^{\frac{3}{4}} u(t)= \frac{1}{\left(1000 \pi^{2}+t^{3}\right)(1+|u(t)|)}\left[e^{-\left(t+\frac{e}{3}\right)}\left(u^{2}+2|u|\right)+e^{-\left(\frac{\pi}{2}+\cos ^{2} \pi t\right)}\left|D_{\frac{1}{2}}^{\frac{1}{2}} u(t)\right|\right. \\
&\left.+e^{-\left(2+\sin ^{2} \pi t\right)}\left|D_{\frac{1}{2}, \frac{2}{3}}^{5} u(t)\right|\right], \quad t \in I_{\frac{1}{2}, \frac{2}{3}}^{I^{\frac{1}{2}}}, \\
& \frac{1}{10 e} u(5)+\frac{1}{20 \pi} D_{\frac{1}{2}}^{\frac{1}{5}} u(5)=\sum_{i=0}^{\infty} \frac{C_{i}\left|u\left(t_{i}\right)\right|}{1+\left|u\left(t_{i}\right)\right|}, \quad t_{i}=10\left(\frac{1}{2}\right)^{i}, \\
& \frac{1}{20 e} u(10)+\frac{1}{10 \pi} D_{\frac{1}{2}, \frac{2}{3}}^{\frac{1}{5}} u(10)=\sum_{i=0}^{\infty} \frac{D_{i}\left|u\left(t_{i}\right)\right|}{1+\left|u\left(t_{i}\right)\right|}, \quad t_{i}=10\left(\frac{1}{2}\right)^{i}+\frac{2}{3}[i]_{\frac{1}{2}},
\end{aligned}
$$

where $C_{i}, D_{i}$ are given constants with $\frac{1}{2000 e^{2}} \leq \sum_{i=0}^{\infty} C_{i} \leq \frac{1}{2000 e}$ and $\frac{1}{1000 \pi^{3}} \leq \sum_{i=0}^{\infty} D_{i} \leq$ $\frac{1}{1000 \pi^{2}}$.

We let $\alpha=\frac{1}{3}, \beta=\frac{3}{4}, \gamma=\frac{1}{5}, \theta=\frac{1}{2}, \nu=\frac{2}{5}, q=\frac{1}{2}, \omega=\frac{2}{3}, \omega_{0}=\frac{\omega}{1-q}=\frac{4}{3}, T=10, \eta=5, \lambda_{1}=\frac{1}{10 e}$, $\lambda_{2}=\frac{1}{20 \pi}, \mu_{1}=\frac{1}{20 e}, \mu_{2}=\frac{1}{10 \pi}$, and

$$
\begin{aligned}
F\left[t, u(t), D_{q}^{\theta} u(t), D_{q, \omega}^{v} u(t)\right]= & \frac{1}{\left(1000 \pi^{2}+t^{3}\right)(1+|u(t)|)}\left[e^{-\left(t+\frac{e}{3}\right)}\left(u^{2}+2|u|\right)\right. \\
& \left.+e^{-\left(\frac{\pi}{2}+\cos ^{2} \pi t\right)}\left|D_{\frac{1}{2}}^{\frac{1}{2}} u(t)\right|+e^{-\left(2+\sin ^{2} \pi t\right)}\left|D_{\frac{1}{2}, \frac{2}{3}}^{\frac{2}{5}} u(t)\right|\right] .
\end{aligned}
$$

We find that

$$
\left|\mathbf{A}_{\eta}\right|=0.308, \quad\left|\mathbf{A}_{T}\right| \approx 0.0207, \quad\left|\mathbf{B}_{\eta}\right| \approx 0.0723, \quad\left|\mathbf{B}_{T}\right| \approx 0.0308,
$$

and

$$
|\Omega| \approx 0.00799
$$

For all $t \in[0,10]$ and $u, v \in \mathbb{R}$, we find that

$$
\begin{aligned}
& \left|F\left[t, u, D_{q}^{\theta} u, D_{q, \omega}^{v} u\right]-F\left[t, v, D_{q}^{\theta} v, D_{q, \omega}^{v} v\right]\right| \\
& \quad \leq \frac{1}{1000 \pi^{2} e^{\frac{e}{3}}}|u-v|+\frac{1}{1000 \pi^{2} e^{\frac{\pi}{2}}}\left|D_{q}^{\theta} u-D_{q}^{\theta} v\right|+\frac{1}{1000 \pi^{2} e^{2}}\left|D_{q, \omega}^{v} u-D_{q, \omega}^{v} v\right| .
\end{aligned}
$$

Thus, $\left(H_{1}\right)$ holds with $\ell_{1}=0.0000409, \ell_{2}=0.0000211$, and $\ell_{3}=0.0000137$. 
For all $u, v \in \mathcal{C}$,

$$
\begin{aligned}
& \left|\phi_{1}(u)-\phi_{1}(v)\right| \leq \frac{1}{2000 e}\|u-v\|_{\mathcal{C}}, \\
& \left|\phi_{2}(u)-\phi_{2}(v)\right| \leq \frac{1}{1000 \pi^{2}}\|u-v\|_{\mathcal{C}} .
\end{aligned}
$$

Thus, $\left(H_{2}\right)$ holds with $\xi_{1}=0.000184$ and $\xi_{2}=0.000101$.

From

$$
\mathcal{O}_{1}=0.2778, \quad \mathcal{O}_{2}=0.2898, \quad \Upsilon_{1}=2.2278, \quad \Upsilon_{2}=0.5169
$$

and

$$
\Theta=1.0549,
$$

we find that $\left(H_{3}\right)$ holds with

$$
\mathcal{X} \approx 0.000542<\frac{1}{3}
$$

Hence, by Theorem 3.1 problem (5.1) has a unique solution.

\section{Conclusion}

A nonlocal Robin boundary value problem for fractional sequential fractional Hahn- $q$ equation (1.1) is studied. Our problem contains both fractional Hahn and fractional qdifference operators, which is a new idea. We establish the conditions for the existence and uniqueness of solution for problem (1.1) by using the Banach fixed point theorem, and the conditions of at least one solution by using Schauder's fixed point theorem.

Acknowledgements

This research was supported by Chiang Mai University.

Funding

This research was funded by King Mongkut's University of Technology North Bangkok. Contract no. KMUTNB-61-GOV-D-64.

Availability of data and materials

Not applicable.

Ethics approval and consent to participate

Not applicable.

Competing interests

The authors declare that they have no competing interests.

Consent for publication

Not applicable.

Authors' contributions

All authors contributed equally to this work. All authors read and approved the final manuscript.

\section{Author details}

${ }^{1}$ Research Center in Mathematics and Applied Mathematics, Department of Mathematics, Faculty of Science, Chiang Mai University, Chiang Mai, Thailand. ${ }^{2}$ Department of Mathematics, Faculty of Applied Science, King Mongkut's University of Technology North Bangkok, Bangkok, Thailand. ${ }^{3}$ Mathematics Department, Faculty of Science and Technology, Suan Dusit University, Bangkok, Thailand. 


\section{Publisher's Note}

Springer Nature remains neutral with regard to jurisdictional claims in published maps and institutional affiliations.

\section{Received: 23 April 2020 Accepted: 7 June 2020 Published online: 15 June 2020}

\section{References}

1. Jackson, F.H.: On q-difference equations. Am. J. Math. 32, 305-314 (1910)

2. Carmichael, R.D.: The general theory of linear q-difference equations. Am. J. Math. 34, 147-168 (1912)

3. Mason, T.E.: On properties of the solutions of linear $q$-difference equations with entire function coefficients. Am. J. Math. 37, 439-444 (1915)

4. Hamza, A.E., Sarhan, A.M., Shehata, E.M., Aldwoah, K.A.: A general quantum difference calculus. Adv. Differ. Equ. 2015, Article ID 182 (2015)

5. Al-Salam, W.A.: Some fractional $q$-integrals and q-derivatives. Proc. Edinb. Math. Soc. 15(2), 135-140 (1966/1967)

6. Agarwal, R.P.: Certain fractional $q$-integrals and q-derivatives. Proc. Camb. Philos. Soc. 66, 365-370 (1969)

7. Kac, V., Cheung, P.: Quantum Calculus. Springer, New York (2002)

8. Goodrich, C.S., Peterson, A.C.: Discrete Fractional Calculus. Springer, New York (2015)

9. Annaby, M.H., Mansour, Z.S.: q-Fractional Calculus and Equations. Lecture Notes in Mathematics, vol. 2056. Springer, Berlin (2012)

10. Rajković, P., Marinković, S., Stanković, M.: Fractional integrals and derivatives in q-calculus. Appl. Anal. Discrete Math. 1(1), 311-323 (2007)

11. Ahmad, B., Ntouyas, S.K.: Boundary value problems for $q$-difference inclusions. Abstr. Appl. Anal. 2011, Article ID $292860(2011)$

12. Ahmad, B., Nieto, J.J.: On nonlocal boundary value problems of nonlinear $q$-difference equations. Adv. Differ. Equ. 2012, Article ID 81 (2012)

13. Ahmad, B., Ntouyas, S.K.: Existence of solutions for nonlinear fractional $q$-difference inclusions with nonlocal Robin (separated) conditions. Mediterr. J. Math. 10, 1333-1351 (2013)

14. Agarwal, R.P., Wang, G., Hobiny, A., Zhang, L., Ahmad, B.: Existence and nonexistence of solutions for nonlinear second order q-integro-difference equations with non-separated boundary conditions. J. Nonlinear Sci. Appl. 8, 976-985 (2015)

15. Ahmad, B., Nieto, J.J., Alsaedi, A., Al-Hutami, H.: Existence of solutions for nonlinear fractional $q$-difference integral equations with two fractional orders and nonlocal four-point boundary conditions. J. Franklin Inst. 351, 2890-2909 (2014)

16. Almeida, R., Martins, N.: Existence results for fractional $q$-difference equations of order $\alpha \in] 2,3$ [ with three-point boundary conditions. Commun. Nonlinear Sci. Numer. Simul. 19, 1675-1685 (2014)

17. Abdeljawad, T., Benli, B., Baleanu, D.: Generalized q-Mittag-Leffler function by $q$-Caputo fractional linear equations. Abstr. Appl. Anal. 2012, Article ID 546062 (2012)

18. Baleanu, D., Agarwal, P.: Certain inequalities involving the fractional-integral operators. Abstr. Appl. Anal. 2014, Article ID 371274 (2014)

19. Zhao, Y., Chen, H., Zhang, Q.: Existence results for fractional $q$-difference equations with nonlocal $q$-integral boundary conditions. Adv. Differ. Equ. 2013, Article ID 48 (2013)

20. Ferreira, R.A.: Nontrivial solutions for fractional $q$-difference boundary value problems. Electron. J. Qual. Theory Differ. Equ. 2010, Article ID 70 (2010)

21. Pongarm, N., Asawasamrit, S., Tariboon, J., Ntoyas, S.K.: Multi-strip fractional q-integral boundary value problems for nonlinear fractional $q$-difference equations. Adv. Differ. Equ. 2014, Article ID 13 (2014)

22. Sitthiwirattham, T., Tariboon, J., Ntouyas, S.K.: Three-point boundary value problems of nonlinear second-order q-difference equations involving different numbers of q. J. Appl. Math. 2013, Article ID 763786 (2013)

23. Saengngammongkhol, T., Kaewwisetkul, B., Sitthiwirattham, T.: Existence results for nonlinear second-order q-difference equations with q-integral boundary conditions. Differ. Equ. Appl. 7(3), 303-311 (2015)

24. Sitthiwirattham, T:: On nonlocal fractional $q$-integral boundary value problems of fractional $q$-difference and fractional $q$-integrodifference equations involving different numbers of order and $q$. Bound. Value Probl. 2016, Article ID 12 (2016)

25. Patanaraprrlert, N., Sitthiwirattham, T.: Existence results of sequential derivatives of nonlinear quantum difference equations with a new class of three-point boundary value problems conditions. J. Comput. Anal. Appl. 18, 844-856 (2015)

26. Patanarapeelert, N., Sriphanomwan, U., Sitthiwirattham, T.: On a class of sequential fractional $q$-integrodifference boundary value problems involving different numbers of $q$ in derivatives and integrals. Adv. Differ. Equ. 2016, Article ID 148 (2016)

27. Sriphanomwan, U., Tariboon, J., Patanarapeelert, N., Sitthiwirattham, T.: Existence results of nonlocal boundary value problems for nonlinear fractional $q$-integrodifference equations. J. Nonlinear Funct. Anal. 2017, Article ID 28 (2017)

28. Patanarapeelert, N., Sitthiwirattham, T.: On four-point fractional q-integrodifference boundary value problems involving separate nonlinearity and arbitrary fractional order. Bound. Value Probl. 2018, Article ID 41 (2018)

29. Etemad, S., Ettefagh, M., Rezapour, S.H.: On the existence of solutions for nonlinear fractional $q$-difference equations with q-integral boundary conditions. J. Adv. Math. Stud. 8(2), 265-285 (2015)

30. Ahmad, B., Etemad, S., Ettefagh, M., Rezapour, S.: On the existence of solutions for fractional q-difference inclusions with q-antiperiodic boundary conditions. Bull. Math. Soc. Sci. Math. Roum. 59(107)(2), 119-134 (2016)

31. Etemad, S., Ntouyas, S.K.: Application of the fixed point theorems on the existence of solutions for 9 -fractional boundary value problems. AlMS Math. 4(3), 997-1018 (2019)

32. Etemad, S., Ntouyas, S.K., Ahmad, B.: Existence theory for a fractional $q$-integro-difference equation with $q$-integral boundary conditions of different orders. Mathematics 2019(7), Article ID 659 (2019)

33. Etemad, S., Rezapour, S., Samei, M.E.: $\alpha-\psi$-Contractions and solutions of a $q$-fractional differential inclusion with three-point boundary value conditions via computational results. Adv. Differ. Equ. 2020, Article ID 218 (2020)

34. Hahn, W.: Über Orthogonalpolynome, die q-Differenzenlgleichungen genügen. Math. Nachr. 2, 4-34 (1949) 
35. Costas-Santos, R.S., Marcellán, F.: Second structure relation for q-semiclassical polynomials of the Hahn tableau. J. Math. Anal. Appl. 329, 206-228 (2007)

36. Kwon, K.H., Lee, D.W., Park, S.B., Yoo, B.H.: Hahn class orthogonal polynomials. Kyungpook Math. J. 38, 259-281 (1998)

37. Foupouagnigni, M.: Laguerre-Hahn orthogonal polynomials with respect to the Hahn operator: fourth-order difference equation for the rth associated and the Laguerre-Freud equations recurrence coefficients. Ph.D. thesis, Université Nationale du Bénin, Bénin (1998)

38. Aldwoah, K.A.: Generalized time scales and associated difference equations. Ph.D. thesis, Cairo University (2009)

39. Annaby, M.H., Hamza, A.E., Aldwoah, K.A.: Hahn difference operator and associated Jackson-Nörlund integrals. J. Optim. Theory Appl. 154, 133-153 (2012)

40. Malinowska, A.B., Torres, D.F.M.: The Hahn quantum variational calculus. J. Optim. Theory Appl. 147, 419-442 (2010)

41. Malinowska, A.B., Torres, D.F.M.: Quantum Variational Calculus. SpringerBriefs in Electrical and Computer Engineering - Control, Automation and Robotics. Springer, Berlin (2014)

42. Malinowska, A.B., Martins, N.: Generalized transversality conditions for the Hahn quantum variational calculus, Optimization 62(3), 323-344 (2013)

43. Hamza, A.E., Ahmed, S.M.: Theory of linear Hahn difference equations. J. Adv. Math. 4(2), 441-461 (2013)

44. Hamza, A.E., Ahmed, S.M.: Existence and uniqueness of solutions of Hahn difference equations. Adv. Differ. Equ. 2013, Article ID 316 (2013)

45. Hamza, A.E., Makharesh, S.D.: Leibniz' rule and Fubinis theorem associated with Hahn difference operator. J. Adv. Math. 12(6), 6335-6345 (2016)

46. Sitthiwirattham, T:: On a nonlocal boundary value problem for nonlinear second-order Hahn difference equation with two different q, $\omega$-derivatives. Adv. Differ. Equ. 2016, Article ID 116 (2016)

47. Sriphanomwan, U., Tariboon, J., Patanarapeelert, N., Ntouyas, S.K., Sitthiwirattham, T.: Nonlocal boundary value problems for second-order nonlinear Hahn integro-difference equations with integral boundary conditions. Adv. Differ. Equ. 2017, Article ID 170 (2017)

48. Brikshavana, T., Sitthiwirattham, T:: On fractional Hahn calculus with the delta operators. Adv. Differ. Equ. 2017, Article ID 354 (2017)

49. Wang, Y., Liu, Y., Hou, C.: New concepts of fractional Hahn's q, $\omega$-derivative of Riemann-Liouville type and Caputo type and applications. Adv. Differ. Equ. 2018, Article ID 292 (2018)

50. Patanarapeelert, N., Sitthiwirattham, T.: Existence results for fractional Hahn difference and fractional Hahn integral boundary value problems. Discrete Dyn. Nat. Soc. 2017, Article ID 7895186 (2017)

51. Patanarapeelert, N., Brikshavana, T., Sitthiwirattham, T:: On nonlocal Dirichlet boundary value problem for sequential Caputo fractional Hahn integrodifference equations. Bound. Value Probl. 2018, Article ID 6 (2018)

52. Patanarapeelert, N., Sitthiwirattham, T.: On nonlocal Robin boundary value problems for Riemann-Liouville fractional Hahn integrodifference equation. Bound. Value Probl. 2018, Article ID 46 (2018)

53. Dumrongpokaphan, T., Patanarapeelert, N., Sitthiwirattham, T.: Existence results of a coupled system of Caputo fractional Hahn difference equations with nonlocal fractional Hahn integral boundary value conditions. Mathematics 2019(7), Article ID 15 (2019)

54. Khan, H., Tunç, C., Alkhazan, A., Ameen, B., Khan, A.: A generalization of Minkowski's inequality by Hahn integral operator. J. Taibah Univ. Sci. 12, 506-513 (2018)

55. Tariboon, J., Ntouyas, S.K., Sutthasin, B.: Impulsive fractional quantum Hahn difference boundary value problems. Adv. Differ. Equ. 2019, Article ID 220 (2019)

56. Sitho, S., Sudprasert, C., Ntouyas, S.K., Tariboon, J.: Noninstantaneous impulsive fractional quantum Hahn integro-difference boundary value problems. Mathematics 8, Article ID 671 (2020)

57. Dumrongpokaphan, T., Patanarapeelert, N., Sitthiwirattham, T.: On sequential fractional q-Hahn integrodifference equations. Mathematics 8, Article ID 753 (2020)

58. Bohner, M., Peterson, A.C.: Dynamic Equations on Time Scale: An Introduction with Applications. Birkhäuser, Boston (2001)

59. Griffel, D.H.: Applied Functional Analysis. Ellis Horwood, Chichester (1981)

60. Guo, D., Lakshmikantham, V.: Nonlinear Problems in Abstract Cone. Academic Press, Orlando (1988)

\section{Submit your manuscript to a SpringerOpen ${ }^{\circ}$ journal and benefit from:}

- Convenient online submission

- Rigorous peer review

- Open access: articles freely available online

- High visibility within the field

- Retaining the copyright to your article

Submit your next manuscript at $\gg$ springeropen.com 